\title{
Implication of Microbial Biofilm in the Biodeterioration of Cementitious Materials in the specific context of Anaerobic Digestion Conditions
}

\author{
Cédric Perez ${ }^{1}$, Christine Lors ${ }^{2}$, and Benjamin Erable ${ }^{1}$ \\ ${ }^{1}$ Laboratoire de Génie Chimique (LGC), CNRS, Université de Toulouse, 31432 Toulouse, France, \\ cedric.perez@ensiacet.fr \\ ${ }^{2}$ Laboratoire de Génie Civil et Géoenvironnement (LGCgE), IMT Lille Douai, Université de \\ Lille, EA 4515, CERI Matériaux \& Procédés, 59500 Douai, France
}

\begin{abstract}
Anaerobic digestion is a renewable energy production process based on the fermentation of biodegradable biomass. The industrial digesters are usually made of cementitious materials. However, the microbial production of several aggressive compounds $\left(\mathrm{CO}_{2}, \mathrm{NH}_{4}^{+}\right.$and volatile fatty acids) during the digestion leads to the deterioration of the concrete structure. The growth of microbial biofilm on the surface of concrete is suspected to generate an even more severe biodeterioration. The goal of this study is to provide a better understanding of the biofilm involvement in the biodeterioration of cementitious materials during an anaerobic digestion process. More precisely, this study is focused on the biofilm heterogeneity and its development on cementitious materials in anaerobic digestion. Lab scale anaerobic bioreactors mimicking industrial anaerobic digestion medium were carried out to immerge CEM I cement pastes in this medium during 2, 3, 4 and 5 weeks. The deterioration of cement pastes was evaluated by using a scanning electron microscope to determine the deteriorated thickness and to quantify the volatile fatty acids in the medium. Biofilm attached on the surface of cement pastes was analyzed through molecular biology techniques, such as 16s rRNA gene sequencing analysis and qPCR.To assess the biofilm heterogeneity, successive stalls of the layers of the biofilm were realized using physical biofilm removal techniques. Three microbial fractions are defined: the planktonic microorganisms, the lousy attached and the strongly attached ones. Results showed that the methanogenic Archaea are found mainly in the medium while around half of the microbial population strongly attached is made of acidogenic bacteria. These results suggest that the biofilm could increase the biodétérioration of concrete since the fatty acids are massively produced at the proximity of the surface of the cementitious samples.
\end{abstract}

Keywords: Biodeterioration, Cementitious Material, Biofouling, Biofilm, Bacteria, Anaerobic Digestion. 


\section{Introduction}

Anaerobic digestion is a renewable energy production process based on the fermentation of biomass. It consists in the succession of four microbial reactions: hydrolysis, acidogenesis, acetogenesis and methanogenesis, leading to the transformation of the biomass into two valuable interest products: the biogas and the digestate. The biogas is mainly constituted of $\mathrm{CH}_{4}$ and $\mathrm{CO}_{2}$ at a concentration of about $40-75 \%$ of $\mathrm{CH}_{4}$. The digestate can be used as a fertilizer for agronomic purposes (Bharathiraja et al., 2018).

In the current period of energy transition towards non fossil renewable energies, the anaerobic digestion process shows a growing interest (Salvador et al., 2019), especially on aspects of improving production performance and enriching $\mathrm{CH}_{4}$ level. In this respect, the focus is on tracks that could increase its durability and thus its performance. One of those tracks could be the durability of the anaerobic digestion tanks (digesters) since it is linked to their lifetime and costs of maintenance. Industrials anaerobic digestion tanks are usually made out of concrete as it is a low cost material, easy to use and adapted to several $\mathrm{m}^{3}$ tanks. However, anaerobic digestion media is known for being aggressive towards the cementitious materials. The microbial metabolism produces aggressive compounds $\left(\mathrm{CO}_{2}, \mathrm{NH}_{4}\right.$, volatile fatty acids), which deteriorate the cementitious matrix. In addition, the formation of microbial biofilms on the concrete surface is highly suspected to increase the kinetics of this deterioration (Voegel et al., 2015,2016). Microbial biofilms can be described as an aggregation of microorganisms on solid surfaces embedded in a self-produced extracellular matrix; called EPS (extracellular polymeric substances). This microbial organization in biofilms allows the formation of microenvironments locally within the biofilm, as a result of the heterogenic repartition of the different

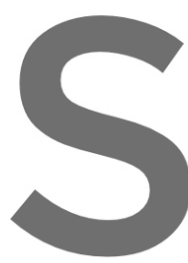
microbial communities, the deterioration mech identified, there is a conditions locally on interface between the micr Magniont et al., 2011)
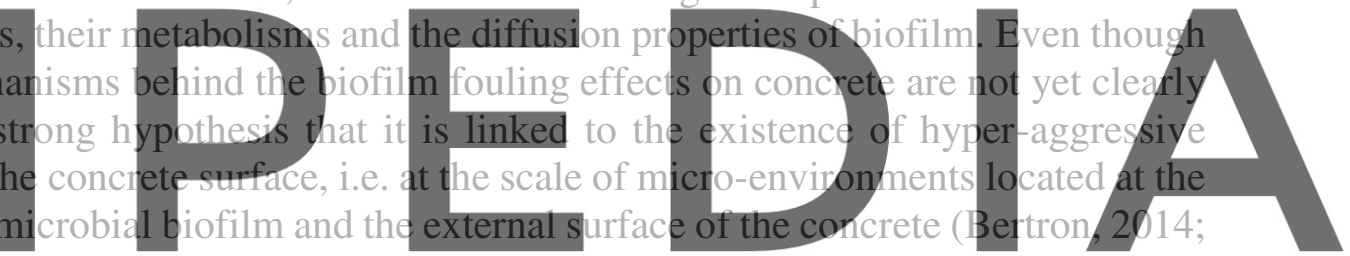

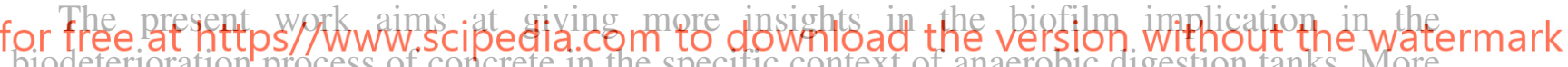
biodeterioration process of concrete in the specific context of anaerobic digestion tanks. More precisely, this study is focused on the visualization and kinetics monitoring of the development of anaerobic digestion biofilms on cementitious materials, and the exploration of bacterial population heterogeneity in the different biofilm layers.

\section{Material and Methods}

\subsection{Cement Paste Pellets Fabrication}

Ordinary CEM I cement pastes were made with a water/cement mass ratio of 0.30 . Cylindrical moulds of $2.8 \mathrm{~cm}$ of diameter and $6.5 \mathrm{~cm}$ height were used. A plastic straw was inserted inside the cement paste at the cylinder central axis, in order to leave an empty space necessary to suspend the paste in the medium during the biodeterioration assays. The cement pastes were then cured for 28 days at $20^{\circ} \mathrm{C}$ inside their closed moulds. After curing, cement paste pellets with a thickness of about $2.5 \mathrm{~mm}$ were obtained by cutting under water the cylindrical cement paste with a circular saw (Prezi, Mecatom 180). The pellets were then polished with a silicon carbide polishing wheel (ESCIL, P800-22 $\mu \mathrm{m}$ ). 


\subsection{Microbial Inoculum and Anaerobic Digestion Culture Medium}

Both microbial inoculum and culture medium were chosen to mimic industrial anaerobic digestion, according to the protocol and methods already described by (Voegel et al., 2016). The medium was a synthetic biowaste made up of different organic fractions which have been blended for $5 \mathrm{~min}$ at $20^{\circ} \mathrm{C}$ (Table 1). The inoculum used was an activated sludge sampled from a municipal wastewater treatment plant in Toulouse (France). The volumic ratio microbial inoculum/medium was $2.5 / 1$.

Table 1. Composition of the synthetic biowaste.

\begin{tabular}{cc}
\hline Organic fractions & Mass $(\%)$ \\
\hline Water & 75.6 \\
\hline potatoes & 8.1 \\
\hline tomatoes & 3.4 \\
\hline Minced meat & 8.1 \\
\hline Milk powder & 0.7 \\
Crackers & 4.1 \\
\hline
\end{tabular}

\subsection{Anaerobic Biodeterioration Assays}

The protocol of the cement paste immersion in the culture medium is made to mimic the operating conditions of anaerobic digestion (Voegel et al., 2016). The immersion of the cements pastes pellets were tested in two types of reactors: type A and type B reactors containing

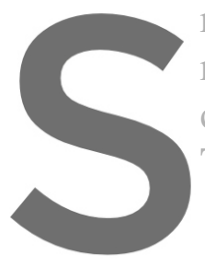
respectively eight pelle reactors contained 52 cement paste and the volu These reactors were car

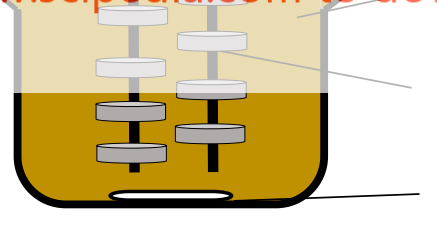

Type A

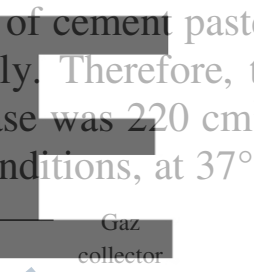

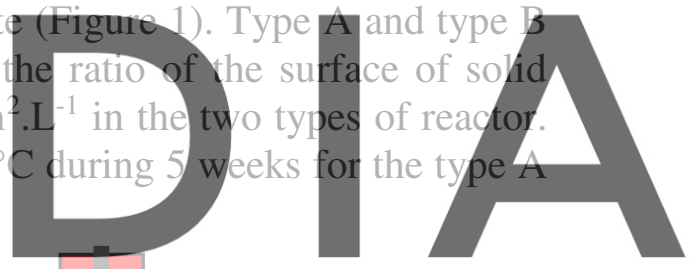

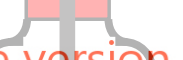

without the watermark

Figure 1. Experimental designs of the anaerobic digestion cultures.

reactor and 2, 3, 4 and 5 weeks for the type $\mathrm{B}$ reactor (triplicates for each duration tested). The gas phase (biogas) was collected continuously in the type A reactor and every day in the type $\mathrm{B}$ reactor with a collection bag connected to a needle.

\subsection{Chemical Analysis}

Samples of the medium were taken at the end of each culture and then filtered at $0.2 \mu \mathrm{m}$. Ammonium ions concentration was measured using Hach kit LCK 304, following manufacturer instructions. Dissolved organic carbon was determined with a TOC analyzer (TOCSHIMADZU Combustion). Acetic, butyric and propionic acids concentrations were measured through high performance liquid chromatography (Thermo Scientific, Accela system, column Rezex ROA H $\mathrm{H}^{+} 8 \%$, eluent $\mathrm{H}_{2} \mathrm{SO}_{4} 10 \mathrm{mM}$, flow rate $170 \mu \mathrm{L} \cdot \mathrm{min}^{-1}$ ). 


\subsection{Microbial Populations Analysis}

Concerning the biofilm sampling on the surface of cement paste pellets, two types of biofilm layers were defined: the "lousy attached" biomass and the "strongly attached" biomass. The "lousy attached" biomass was removed from a biocolonized cement paste pellet through an immersion of the pellet in $3 \mathrm{~mL}$ of phosphate buffered saline (PBS, 0.1M, pH 7.4) for 15 minutes. The "strongly attached" biomass was removed from a biocolonized cement paste pellet with a sonication treatment of 3 minutes in $5 \mathrm{~mL}$ of PBS. Removing treatments were not done successively, thus the "strongly attached" biomass should also contain the "lousy attached" one. $2 \mathrm{~mL}$ of the culture medium were as well sampled in triplicate. The samples of biocolonized cement paste originated from a type A reactor after 5 weeks of culture. Each removal protocol has been performed in triplicate, i.e. starting from three different biocolonized cement pellets. DNA extraction was performed on the three types of samples (liquid, strongly "attached and lousy attached" biomass) obtained using a DNeasy power biofilm kit, according to manufacturer instructions. Sequencing of the 16S SSU RNA was done using 515F and 806R primers targeting both bacteria and Archaea. The sequencing and its statistical analysis were performed by RTlab (USA).

\subsection{Physical Analysis}

Cement paste pellets from the type A reactor were taken out after 2, 3, 4 and 5 weeks. A cut of the cement paste pellets was realized with a circular saw, in order to divide the cylindrical sample into two identical parts. A part of the pellet was then impregnated under vacuum with epoxy resin (Araldite, 2020) in circular molds (diameter $=2.6 \mathrm{~cm}$ ). After hardening of the epoxy

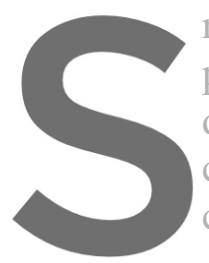
resin, the surface was particle sizes of 200 diamond-covered polis coupled with a EDX detector (Thermo Scientif
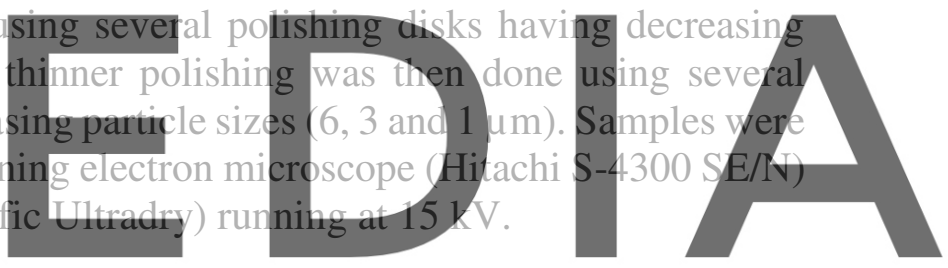


\section{Results and Discussion}

\subsection{Deterioration Profile of the Cement Paste}

The evolution of the concentrations of volatile fatty acids (VFAs), ammonium ions and inorganic carbon from 2 to 5 weeks of anaerobic digestion process are shown on (Figure 2).
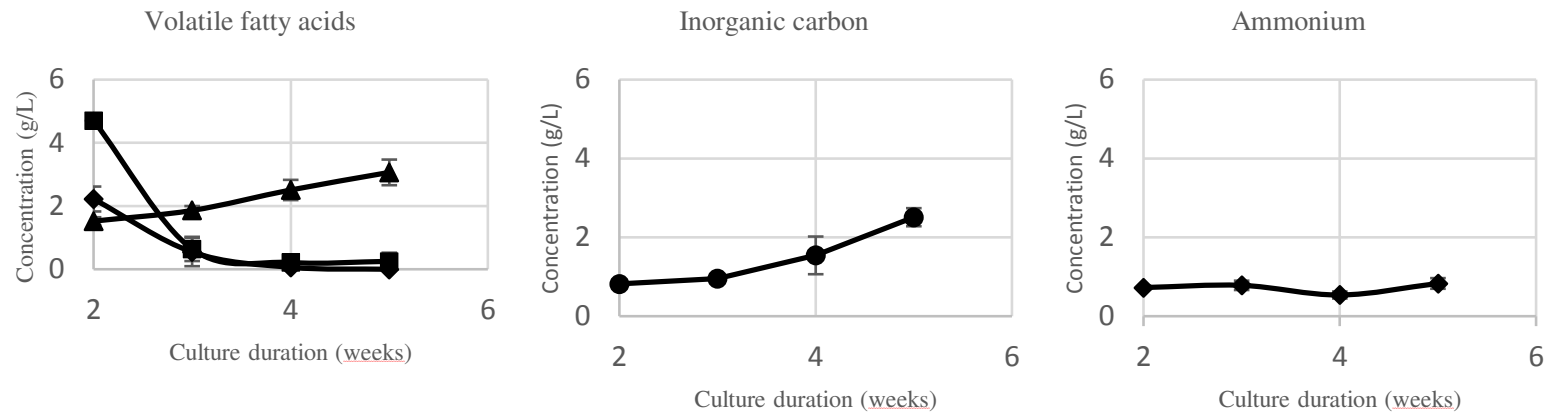

$\longrightarrow$ Ac acétique $\longrightarrow$ Ac propionique

Figure 2. Evolution of concentrations of the aggressive metabolic compounds for a period of 5 weeks of anaerobic digestion process.

After two weeks of anaerobic digestion, the concentration of acetic and butyric acids were of 4.7 g. $\mathrm{L}^{-1}$ and 2.2 g. $\mathrm{L}^{-1}$ respectively. Their concentrations strongly decreased between week 2 and week 3 to finally stabilize around $0 \mathrm{~g} / \mathrm{L}$ at end of the fourth week of anaerobic digestion. The propionic acid concentration evolved differently, since it was still increasing constantly from 1.5 to $3.0 \mathrm{~g} . \mathrm{L}^{-1}$ in the period of time between the $2^{\text {nd }}$ and $t$ After 2 weeks, both ace Voegel et al. (2016), whro

More curiously, the propic
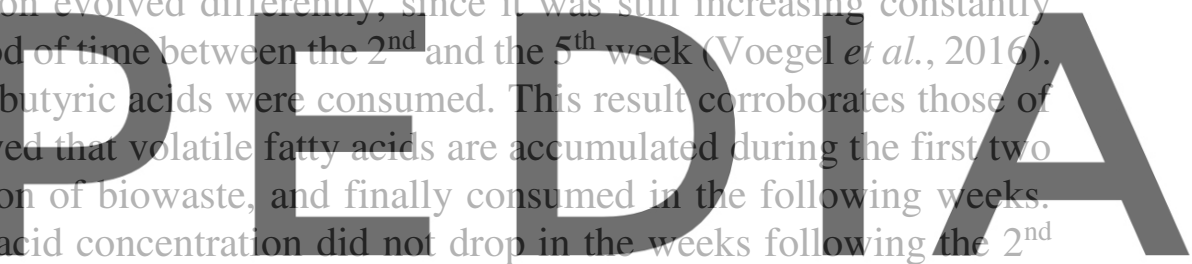

week, suggesting that this VFA has not been reused or metabolized by acetogenic microbial

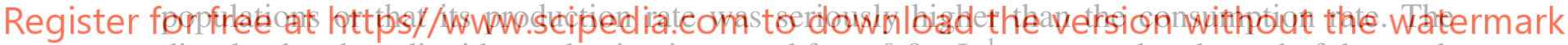
dissolved carbon dioxide production increased from $0.8 \mathrm{~g} \cdot \mathrm{L}^{-1}$, measured at the end of the week 2, to $2.5 \mathrm{~g} . \mathrm{L}^{-1}$ after 5 weeks. The concentration of ammonium has remained very stable, around 0.7 g.L.-1, from week 2 to week 5. Since ammonium ions are byproducts of amino acid fermentation (Ramsay and Pullammanappallil, 2001), it should be produced only during the acidogenesis stage occurring mainly during the first two weeks. In this way, we can make the hypothesis that the aggressive nature of the anaerobic digestion environment was at its maximum during the first two weeks of the experiment, since the concentration of aggressive compounds should be at its peak during this period.

Figure 3 allows the localization of the biodeteriorated zones in the depth of a cement paste sample exposed for 5 weeks in an anaerobic digestion medium. 

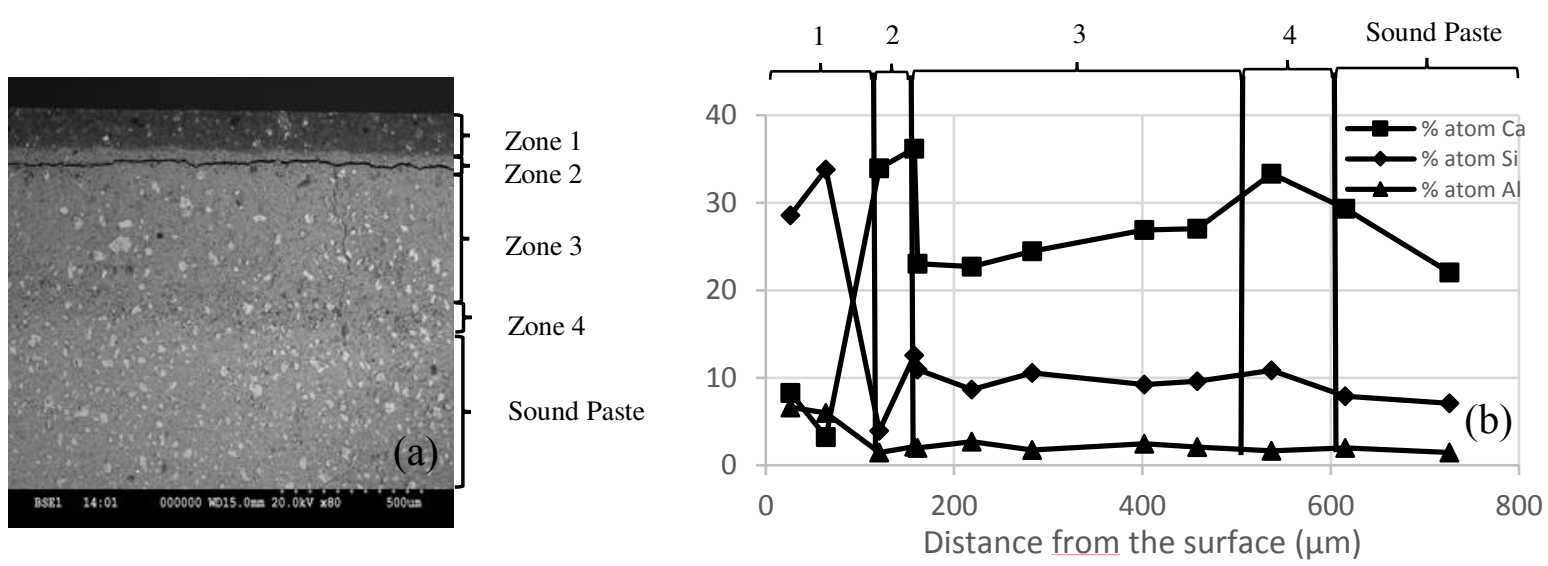

Figure 3. Repartition of the deteriorated zones within the depth of a cement paste (a) and the deterioration profile of a cement paste immerged for 5 weeks in an anaerobic digestion medium (b).

Four zones can be identified (Figure 3): a first layer darker than the others, of about $100 \mu \mathrm{m}$. A second zone, about 20-30 $\mu \mathrm{m}$, can be identified around the crack. The third zone has a variable thickness among the different duration tested from 150 to $400 \mu \mathrm{m}$. A fourth zone darker than the third one is also observed, its thickness also changes from a duration to another from 150 to $430 \mu \mathrm{m}$, and finally at the heart of the cement paste there is a last zone corresponding to the sound paste. Figure 3(b) shows the evolution of the relative proportion of calcium $(\mathrm{Ca})$, silicium (Si) and aluminum (Al) from the outer surface to $800 \mu \mathrm{m}$ in the depth of biodeteriorated

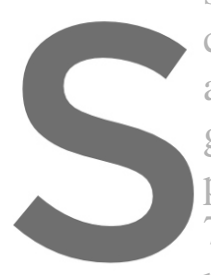
cement paste sample. another. All of those $\mathrm{z}$ global composition in e proportion, around $10 \%$ $7 \%$, in regard to the oth
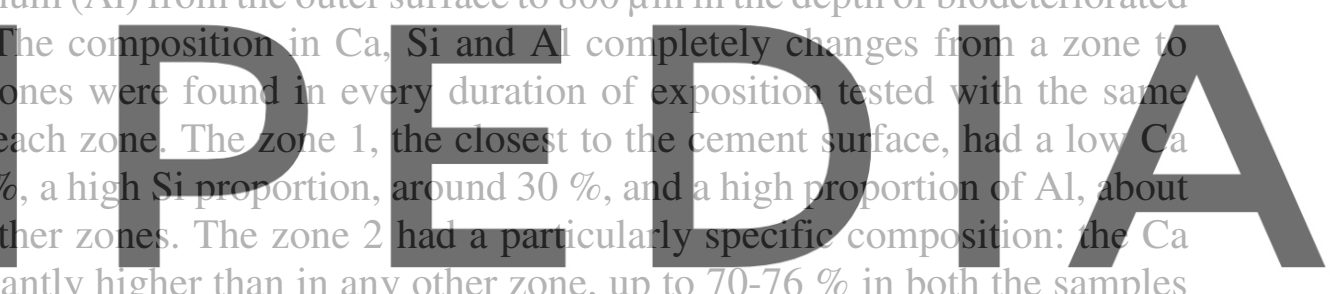

proportion was significantly higher than in any other zone, up to 70-76\% in both the samples

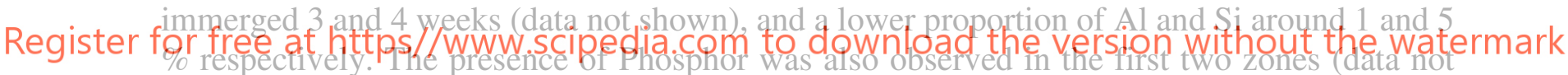
shown). In the three other zones (zones 2, 4 and 5), only a very small divergence in composition was detected: between 20 and $30 \%$ of $\mathrm{Ca}$, around $10 \%$ in average of Si and 1.5 to $2 \%$ of Al. Those results are representative of a $\mathrm{Ca}$ leaching and a $\mathrm{P}$ enrichment in the layers close to the surface. However, chemical element proportion analysis can not precisely show every change in mineral composition, as proportion could remain the same while the quantity could change.

Analysis with other techniques, such as X-Ray Diffraction, could give more information, particularly on the residual anhydrous grains. Those results are in accordance with the observations already reported by (Voegel et al., 2016), who studied also cement paste biodeterioration after exposition to digested biowastes. These authors identified $\mathrm{Ca}$ leaching as part of the biodeterioration mechanisms.

Table 2 shows the evolution of the thickness of the total biodeteriorated zone for increasing time of cement paste exposition to anaerobic digestion environment. The thickness of the total biodeteriorated zone was determined by the Ca cartography obtained by SEM.

Table 2. Evolution thickness of the total biodeteriorated zone with the exposition time in anaerobic digestion medium.

\begin{tabular}{cccc}
\hline Exposition time (weeks) & 2 & 3 & 5 \\
\hline Thickness of the total biodeteriorated zone $(\mu \mathrm{m})$ & 554 & 594 & 619 \\
\hline
\end{tabular}


The thickness of the total biodeteriorated zone increases from 2 weeks to 5 weeks of exposition time, from respectively $554 \mu \mathrm{m}$ to $619 \mu \mathrm{m}$. The same kind of observations on cement pastes exposed for longer period, 10 and 15 weeks, should in the future make it possible to confirm this pattern.

\subsection{Microbial Populations of the Biofilm}

Figure 4 shows the repartition of the different bacterial phyla within the liquid fraction and in the "lousy attached" biomass and the "strongly attached" biomass collected on the surface of a cement paste after 5 weeks in type B reactor. Planktonic and sessile (attached) bacterial populations were different. Indeed, the proportion of Archaea was fairly higher in the liquid fraction, i.e. $22.0 \%$ against $3.4 \%$ in the attached biomass.

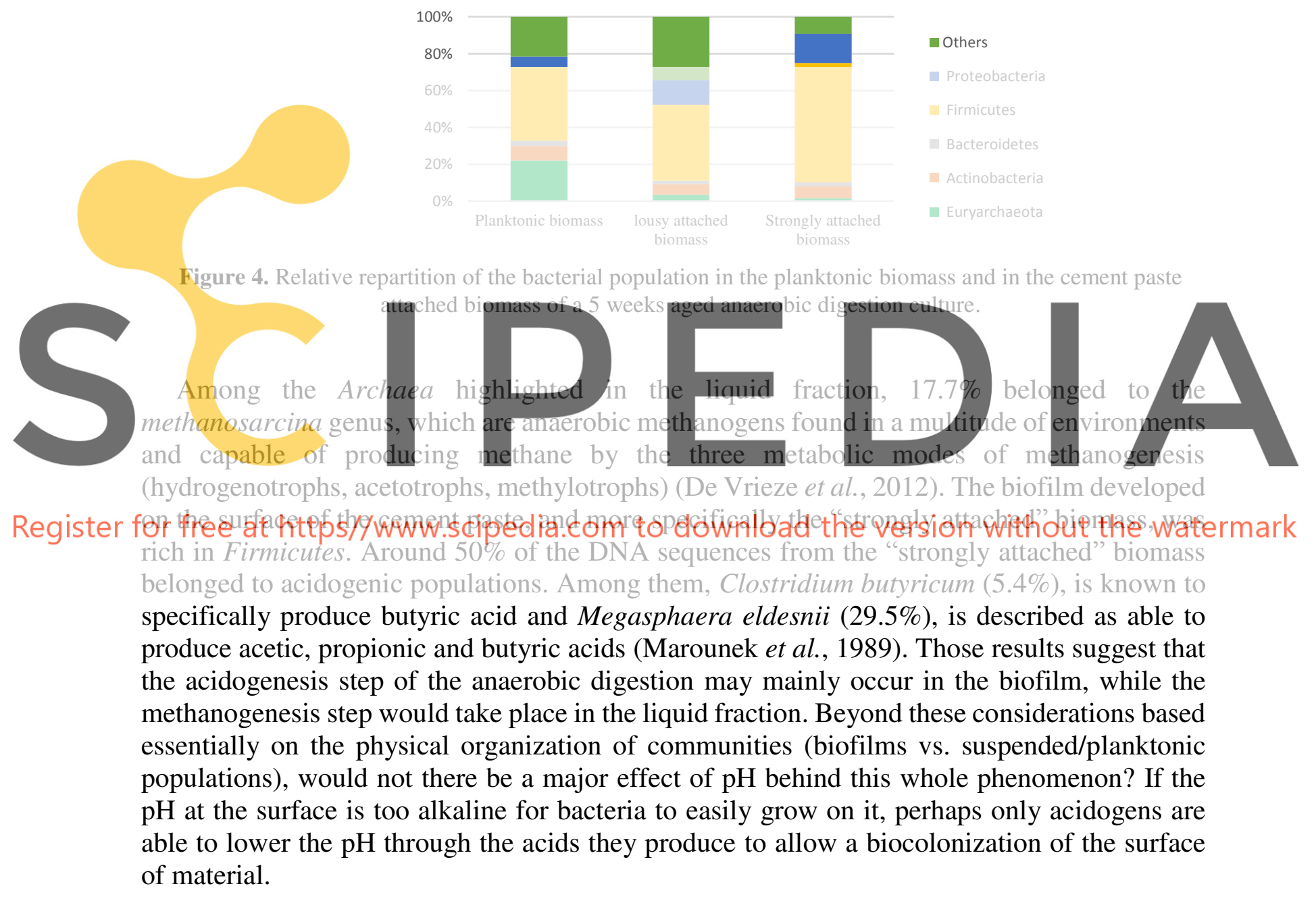




\section{Conclusion}

The SEM analysis of the cement pastes exposed from 2 to 5 weeks to an anaerobic digestion medium showed a biodeterioration of the cement paste characterized by a depth up to $700 \mu \mathrm{m}$ with calcium leaching and phosphor enrichment in the first $100 \mu \mathrm{m}$. Bacteria present in the biofilm colonizing the cement paste are mainly acidogens, which may confirm the hypothesis that the microbial fouling of the cement paste is caused by the local production of aggressive compounds, such as organic acids acting on reducting the surface $\mathrm{pH}$ of cementitious materials. The use of fluorescent dyes could help measure the $\mathrm{pH}$ both in the biofilm and on the surface of the cement paste through fluorescence microscopy observation techniques.

\section{ORCID}

Cédric Perez: https://orcid.org/0000-0002-2057-5958

Christine Lors: https://orcid.org/0000-0002-2222-5556

Benjamin Erable: https://orcid.org/0000-0002-5332-9622

\section{References}

Bertron, A. (2014). Understanding interactions between cementitious materials and microorganisms: a key to sustainable and safe concrete structures in various contexts. Materials and Structures, 47(11), 1787-1806. https://doi.org/10.1617/s11527-014-0433-1

Bharathiraja, B., Sudharsana, T., Jayamuthunagai, J., Praveenkumar, R., Chozhavendhan, S. and Iyyappan, J. (2018). Biogas production - A review on composition, fuel properties, feed stock and principles of anaerobic

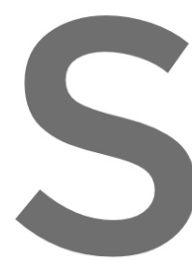
digestion. https:/doi.oro/10.101/jor

De Vrieze, J., Hennebel, for heavy https://doi.org/10.1016/j.thong

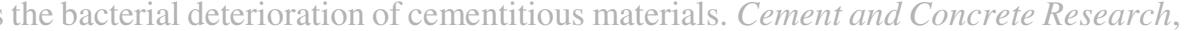

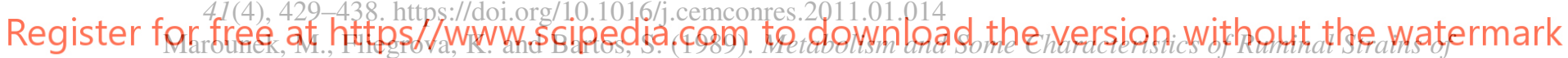
Megasphaera elsdenii. 55, 4.

Ramsay, I. R. and Pullammanappallil, P. C. (2001). Protein degradation during anaerobic wastewater treatment: derivation of stoichiometry. Biodegradation, 12(4), 247-256.

Salvador, R., Barros, M. V., Rosário, J. G. D. P. D., Piekarski, C. M., Luz, L. M. da, and Francisco, A. C. de. (2019). Life cycle assessment of electricity from biogas: A systematic literature review. Environmental Progress \& Sustainable Energy, 38(4). https://doi.org/10.1002/ep.13133

Voegel, C., Bertron, A. and Erable, B. (2015). Biodeterioration of cementitious materials in biogas digester. Matériaux \& Techniques, 103(2), 202. https://doi.org/10.1051/mattech/2015023

Voegel, C., Bertron, A. and Erable, B. (2016). Mechanisms of cementitious material deterioration in biogas digester. Science of The Total Environment, 571, 892-901. https://doi.org/10.1016/j.scitotenv.2016.07.072 